\title{
Photometric survey of the very small near-Earth asteroids with the SALT telescope
}

\section{Lightcurves and periods for 14 objects ${ }^{\star}$}

\author{
T. Kwiatkowski ${ }^{1}$, D. A. H. Buckley ${ }^{2}$, D. O’Donoghue ${ }^{2}$, L. Crause ${ }^{2}$, S. Crawford ${ }^{2}$, Y. Hashimoto ${ }^{2}$, A. Kniazev², \\ N. Loaring ${ }^{2}$, E. Romero Colmenero ${ }^{2}$, R. Sefako ${ }^{2}$, M. Still ${ }^{2}$, and P. Vaisanen ${ }^{2}$ \\ 1 Astronomical Observatory, Adam Mickiewicz University, Słoneczna 36, 60-286 Poznań, Poland \\ e-mail: tkastr@vesta.astro.amu.edu.pl \\ 2 South African Astronomical Observatory, Observatory Road, Observatory 7925, South Africa
}

Received 20 August 2009 / Accepted 2 November 2009

\begin{abstract}
Aims. We report first results from our extensive survey of the very small $(H>21.5 \mathrm{mag})$ near-Earth asteroids. Our aim was to obtain photometric lightcurves for these faint, fast moving objects and to measure their rotation periods and amplitudes of light variations. These parameters can be used to make statistical analysis of the still little known population of the smallest asteroids, test present theories of the YORP effect as well as to study their spin limits, which are connected with their internal structure.

Methods. Due to the faintness of the targets and the expected short periods of rotation, observations were performed with the large, 10-m SALT telescope in SAAO (South Africa). For most asteroids, $V$ filter images with exposure times of 5-60 s were obtained with the instrument's SALTICAM's CCD camera. Even though the non-sidereal tracking was not available, the SALTICAM's relatively large field-of-view of $8^{\prime} \times 8^{\prime}$ helped to perform the relative photometry of the fast-moving targets.

Results. The presented asteroids have synodic periods ranging from $77 \mathrm{~s}$ to 44 min, effective diameters from 21 to 94 m, and significantly elongated shapes.
\end{abstract}

Key words. techniques: photometric - minor planets, asteroids: general

\section{Introduction}

The population of near-Earth asteroids (NEAs) constitutes a sample of the small diameter main-belt asteroids (MBAs) that were redirected towards the inner parts of the solar system. Their orbits have perihelion distances smaller than 1.3 AU. Being relatively close to the Earth, they can easily be studied by optical telescopes, radar, and space probes. Currently (spring 2009), more than 6000 of these objects have been cataloged, but only for 900 of them definite orbits are known ${ }^{1}$.

Observations of NEAs give us access to the smallest diameter objects, which are too faint to be studied in the main belt. Of particular importance here are the asteroids with absolute magnitudes greater than $H=21.5 \mathrm{mag}$ (which corresponds roughly to effective diameters of $D<150 \mathrm{~m}$ ), many of which were found to have very short periods of rotation. While most asteroids rotate with periods longer than a critical value of $2.2 \mathrm{~h}$, the smallest of them can have periods of the order of tens or even single minutes which suggests a significant tensile strength. Examples of such bodies are visible in the Hayabusa photographs of (25 143) Itokawa - a $D=330$ m rubble-pile near-Earth asteroid, whose surface is covered with boulders and stones with diameters of about $D=30 \mathrm{~m}$ down to the size of a pebble (Saito et al. 2006).

\footnotetext{
* Photometric tables are only available in electronic form at the CDS via anonymous ftp to

cdsarc.u-strasbg.fr (130.79.128.5) or via

http://cdsweb.u-strasbg.fr/cgi-bin/qcat?J/A+A/509/A94

${ }^{1}$ http://neo.jpl.nasa.gov/stats/
}

Being very small in size, such boulders are thought of as the building blocks of larger bodies, and their material properties are important for studies of the collisional evolution of asteroids, the formation of binary systems and also for craters on their surfaces. On the other hand, when entering the Earth's atmosphere, such meter-sized asteroids can be also observed as bolides whose fragmentation depends, among all, on their tensile strengths (a good example of such an object was a $D=4 \mathrm{~m} 2008 \mathrm{TC}_{3}$, which ended its life in the Nubian desert; Jenniskens et al. 2009).

The images returned by Hayabusa do not show the internal structure of the boulders populating the surface of Itokawa - they can be either solid or significantly fractured pieces of rocks. However, when rotation periods of individual objects of this type are derived from their lightcurves, it is possible to determine their spin limits, which can then be used to study their tensile strength (Holsapple 2007).

The smallest, rapidly rotating near-Earth asteroids are sometimes called monolithic fast rotating asteroids (MFRAs), but recent studies suggest that not all of them are monoliths (Holsapple 2007). A better term has been used by Pravec et al. (2002), who distinguished a group of small diameter objects $(D<150 \mathrm{~m})$ and called them very small asteroids (VSAs), which refers only to their diameters and does not impose any restrictions on their composition. In this and the following papers we will use the latter nomenclature.

The first asteroids with rotation periods shorter than $2.2 \mathrm{~h}$ were $1995 \mathrm{HM}\left(P=97 \mathrm{~min}\right.$, Steel et al. 1997) and $1998 \mathrm{KY}_{26}$ $(P=10.7 \mathrm{~min}$, Ostro et al. 1999). With a discovery of three new 
such objects (1999 TY $2,1999 \mathrm{SF}_{10}$ and $1998 \mathrm{WB}_{2}$, Pravec et al. $2000)$ it became clear that there exists a population of very small $(H>21.5 \mathrm{mag})$, fast rotating asteroids.

In Pravec et al. (2000) the importance of further studies of these objects was stressed. They defined the directions of research: studies of distributions of spin rates, its dependence on size, taxonomy and tensile strength. It was shown that a future survey can be accomplished with 1-2 m class telescopes, for which objects must be brighter than $V=18$ mag. A list of known, $H<22$ mag objects, discovered during a time span of one year, was presented with the conclusion that roughly half of them were brighter than $V=18 \mathrm{mag}$ after the discovery. One pessimistic aspect of such a survey, according to Pravec et al. (2000) would be that very few of the VSAs would return to the Earth's neighborhood within five years after the discovery to be available for photometric studies. But even for those a rediscovery would be needed as their orbits may not be good enough for accurate ephemerides to be computed.

Whiteley et al. (2002b) discussed the state of research of the VSAs as of 2002. They proposed several ways of extending the database of the spin periods of these objects. One possibility would be to perform a wide field photometric survey of the main belt with an 8-10 m telescope. During such observations many objects from the $H=18-22$ mag transition region could be discovered and their periods measured.

Another interesting problem to be solved, according to Whiteley et al. (2002b), is the lower limit of the rotation period. They noted that the periods of under a minute were not physically implausible. At that time there were small asteroids for which, despite many hours of observations, no brightness changes were detected. That could be caused by a nearly spherical shape, a pole-on aspect or a very long rotation period (many days). It could also be caused by extremely short periods, comparable to the time of the exposures. The latter would smooth out the amplitude to the level of noise.

New results on the rotation of 25 VSAs were presented by Whiteley et al. (2002a), out of which 19 objects had periods shorter than $2.2 \mathrm{~h}$. However, they did not provide any lightcurves or observing circumstances - such data are difficult to be used in further studies except for statistical analysis.

Radar observations of the interesting Earth co-orbital asteroid 2002 AA $_{29}$ (Ostro et al. 2003) allowed only the determination of the upper bound of the asteroid rotation period, which was found to be shorter than $33 \mathrm{~min}$. This is a well known limitation of the radar observing technique and shows how important it is to supplement it with the optical photometry which helps to refine the results.

During the next several years very few new results for fastrotating asteroids were reported in the peer-reviewed literature. In a short abstract Hergenrother \& Whiteley (2005) announced rotation periods for $14 \mathrm{VSAs}$, but the actual numerical results can only be found in the Light Curve Data Base (Warner et al. 2009). The original lightcurves and the observing details are currently not available.

A thorough study of $2004 \mathrm{VD}_{17}$ was presented by de Luise et al. (2007). They combined visible and near-infrared photometry and spectroscopy as well as polarimetric observations to derive the rotation period, diameter, geometric albedo and taxonomic class for this object. Even though $2004 \mathrm{VD}_{17}$ has the effective diameter of $D=320 \mathrm{~m}$, its rotation period of $1.99 \mathrm{~h}$ is close to the threshold value and thus makes it an interesting borderline case.
New rotation periods of VSAs are also reported on the web pages of Behrend ${ }^{2}$ and Pravec ${ }^{3}$ but they often lack detailed descriptions. There is also an ongoing program led by Richard Miles who uses a remotely controlled, 2-m Faulkes Telescope for observations and managed to get periods of several VSAs. One of them, $2008 \mathrm{HJ}$, revealed a period of only $42 \mathrm{~s}$ - the shortest measured so far for an asteroid (Miles 2008).

Recently a new fast-rotating near-Earth asteroid $2001 \mathrm{FE}_{90}$ has been observed (Hicks et al. 2009) for which a period of $P=$ $28 \mathrm{~min}$ was derived. While its effective diameter of $D=200 \mathrm{~m}$ makes it slightly larger than the threshold value for VSAs, this object is unusual because several lightcurves were obtained for it at different positions in the sky which should make it possible to derive its sidereal period, spin axis and shape. $2001 \mathrm{FE}_{90}$ is also one of the few small near-Earth asteroids for which accurate orbits are known. Its good ephemeris will make it possible to observe it again during its next close approach in 2017, when it will brighten to $V=21.7$ mag.

The idea of Whiteley et al. (2002b) of observing VSAs in the main belt was realized by Dermawan (2004), who used a wide-field CCD camera on the 8-m Subaru telescope to obtain lightcurves of many asteroids. As he observed them only on one night, he was unable to determine their orbits, and his objects were effectively lost. The original results of that survey were published in Japanese in a Ph.D. Thesis (Dermawan 2004) and are difficult to access. However, they were recently carefully reviewed by Warner et al. (2009) who found out that many of the rotation periods derived by Dermawan (2004) are uncertain, and thus his claim of finding rapidly rotating asteroids among MBAs has still to be confirmed.

Another attempt at observing fast-rotating asteroids in the main belt was made by Masiero et al. (2009). He found six objects of diameters smaller than $1 \mathrm{~km}$ that rotate with periods shorter than the $2.2 \mathrm{~h}$ threshold. However, due to a significant noise in the lightcurves, their results are also uncertain.

The most complete source of data on asteroid periods is currently the Light Curve Data Base ${ }^{4}$ (LCDB) maintained by Alan Harris, Brian Warner and Petr Pravec (Warner et al. 2009). As of 21 April 2009 it contained 55 asteroids with diameters smaller than $150 \mathrm{~m}$ (with five of them residing in the main belt), 45 of which had rotation periods shorter than $2.2 \mathrm{~h}$ (this statistic includes only asteroids with the quality code $U \geq 2$ ).

To extend our knowledge about the spin properties of the VSAs, in January 2007 we began a systematic, photometric survey of the very small near-Earth asteroids. To reach fainter objects we used a new 10-m Southern African Large Telescope (SALT), located at the South African Astronomical Observatory in the Karoo desert in South Africa. Usually instruments of this aperture are difficult to be used in extended campaigns due to severe time limitations. In the case of SALT, however, it was still in the performance-verification (PV) phase, when not all subsystems worked as expected but that had the advantage that proposals could obtain a substantial amount of time.

The first object from our campaign has been presented in Kwiatkowski et al. (2009). It was an unusual near-Earth asteroid $2006 \mathrm{RH}_{120}$. Here we report lightcurves of 14 VSAs with rotation periods shorter than $2.2 \mathrm{~h}$. The remainder of the results of our survey will be presented in the forthcoming papers.

\footnotetext{
2 http://obswww . unige.ch/ behrend/page_cou.html

3 http://www . asu. cas.cz/ ppravec/neo.htm

4 http://www . minorplanetobserver.com/astlc/

LightcurveParameters.htm
} 


\section{Observations and data reduction}

A detailed description of the SALT telescope and the CCD camera used in our observations was already given in Kwiatkowski et al. (2009) and references therein, so we will mention here only the pertinent details for the present paper.

SALT works similar to the Arecibo radio telescope except that it can rotate its tilted $10-\mathrm{m}$ spherical mirror about the vertical axis prior to the observation. The spherical aberration is corrected by the dedicated optical system mounted, together with the payload, on a movable "tracker" which is located above the main mirror and follows objects during the observation.

Because of this construction, SALT has access to objects in the declination range from $\delta=-75^{\circ}$ to $\delta=+10^{\circ}$, which can be observed when they enter the annular region on the sky with a zenith distance from $31^{\circ}$ to $43^{\circ}$. In most cases objects can be observed during two separate runs on a given night: the "east track" and the "west track", each of which lasts for about an hour. Targets lying close to the telescope declination limits can be observed during single, longer tracks. They are not continuous, however, and are interrupted by the Tracker repositioning.

This basic mode of operation makes SALT a good instrument for observing time-dependent phenomena with periods shorter than about one hour or longer than $24 \mathrm{~h}$ - the former was the case in our survey. As mentioned above, the observations were carried out during the PV phase of SALT, when the telescope was still affected by the limited image quality (IQ). This often resulted in the increased FWHM of stellar images and asymmetric point spread function (PSF). It was not a major problem, however, because we used aperture photometry, and the only drawback from the increased aperture sizes was $S / N$ lower than what could be achieved with the perfectly collimated optics.

Another inconvenience was the stray light from the red autocollimator laser which was used to keep the mirror segments properly adjusted. It affected mainly the clear filter images, though traces could also be noticed when the $V$ filter was used. The scattered light increased the sky level, but was subtracted when the sky background was measured within a circular annulus around the aperture.

During the PV phase SALT did not yet have a moving baffle to compensate for the continuously changing pupil during the observations. As a result it was not possible to correct images for flat-field. We tried to flatten by acquiring the twilight flatfields with the telescope pointing in the same direction (and the CCD camera being rotated at the same angle) as in the middle of the night track. Another attempt was made with the night sky flat-fields, obtained by shifting the field of view (hereafter FoV) slightly between the exposures, then cleaning the stars from the frames and finally stacking the resulting images to increase the $S / N$. In both cases, however, there was little improvement in the obtained photometry (2007 $\mathrm{KE}_{4}$ was an exceptional case here) probably due to the influence of the stray light. The effect of the lack of proper flat-fielding was non-Gaussian noise of the order of about $0.05-0.1 \mathrm{mag}$. As a result, we were unable to unambiguously detect light changes smaller than about $0.1-0.2$ mag.

We should also mention that the autoguider was not yet functional, which made it impossible to use non-sidereal tracking rates and also limited exposure times to $60 \mathrm{~s}$. This was however not a major drawback since we wanted to detect short periods in asteroid lightcurves and exposures longer than one minute would be impractical. Also, with a plate scale of 0.56 per pixel (obtained in a $4 \times 4$ binning mode, which shortened the read-out time to $15 \mathrm{~s}$ ) the night sky saturated the CCD in a "clear" filter in less than $60 \mathrm{~s}$.
Program asteroids were selected based on several criteria: their absolute magnitudes, as reported by the Minor Planet Electronic Circulars (hereafter MPEC), had to be greater than $H=21.5 \mathrm{mag}$, they should be brighter than $V=20.5 \mathrm{mag}$ and obviously they should be accessible to SALT (declinations in the range $-75^{\circ}<\delta<+10^{\circ}$ ). Since at the time of observations the non-sidereal tracking was not available, we also had to limit objects to those which did not move more than 1".5 during a single exposure to avoid significant elongation of their images.

All observations reported here were executed in a queue mode. The obtained data were pre-reduced on site and made available for download, usually a day or two after the observations. More details about the data reduction can be found in Kwiatkowski et al. (2009).

\section{Determination of periods and shapes}

If we assume that during observations the asteroid does not change its position with respect to the observer and the Sun and also that its spin axis remains fixed in space, then its light variations can be approximated by a Fourier series depending on a time $t$ and the period of rotation $P$ (Pravec et al. 2000):

$V(t)=\bar{V}+\sum_{k=1}^{n}\left(A_{k} \sin \frac{2 \pi k\left(t-t_{\mathrm{o}}\right)}{P}+B_{k} \cos \frac{2 \pi k\left(t-t_{\mathrm{o}}\right)}{P}\right)$

where $\bar{V}$ is the average brightness and $A_{k}$ and $B_{k}$ are Fourier coefficients. Depending on the lightcurve shape and its noise this series is truncated at $n=4-8$ with the highest signal usually present in the second order - this reflects a typical two maxima, two minima lightcurve. In the case of fast rotating asteroids, the signal integration time can approach the period itself, smoothing out the fitted Fourier harmonics. This effect has been discussed by Pravec et al. (2000), who derived the equation to compute the scaling factor $f_{k}$ for the Fourier harmonic of the order $k$. In our observations we tried to use as short exposure times as possible to rule out the smoothing of the lightcurve amplitudes. It appeared we were quite successful at that - only on 12 Jan. 2007, when 2006 XY was observed, the exposure time of $60 \mathrm{~s}$ resulted in the second harmonic being $f_{2}=0.75$ of its original value. On the following nights, however, the exposure time was shortened to $30 \mathrm{~s}$ and the scaling factor rose to $f_{2}=0.94$ which, given the overall noise in the lightcurve, need not to be corrected for.

To derive asteroid periods from their lightcurves we solved Eq. (1) by least squares with the assumed period $P$. This procedure was repeated for different periods from a wide interval. At each step a $\chi^{2}$ value was derived and a global minimum was located. In order to estimate the uncertainty of the data points we rescaled the smallest $\chi^{2}$ per degree of freedom to unity and solved for $\sigma$. A detailed description of the algorithm is presented in Kwiatkowski et al. (2009).

Since in reality the assumption of the unchanging geometry of observation does not hold, the measured period is synodic rather then sidereal. Not knowing the asteroid pole position it is not possible to translate the obtained synodic period into a sidereal one. Still, we can estimate the difference between the two using the approximate relation derived by Pravec et al. (2005):

$\Delta P_{\text {syn-sid }} \approx \frac{\mathrm{d}(P A B)}{\mathrm{d} t} P^{2}$,

where $P A B$ is the phase angle bisector and $P$ is the sidereal period (with the assumption of a principal axis rotation). If we use this value as an upper bound of the actual $P_{\text {syn }}-P_{\text {sid }}$ difference 
Table 1. Aspect data and the observing log.

\begin{tabular}{|c|c|c|c|c|c|c|c|c|c|c|c|c|c|}
\hline Asteroid & Date & $\begin{array}{l}\text { Obs. time } \\
\text { (UTC) }\end{array}$ & $\begin{array}{c}r \\
(\mathrm{AU})\end{array}$ & $\begin{array}{c}\Delta \\
(\mathrm{AU})\end{array}$ & $\begin{array}{c}\alpha \\
\left({ }^{\circ}\right)\end{array}$ & $\begin{array}{c}\lambda \\
\left(^{\circ}\right)\end{array}$ & $\begin{array}{c}\beta \\
\left({ }^{\circ}\right)\end{array}$ & $\begin{array}{c}V \\
{[\mathrm{mag}]}\end{array}$ & $\begin{array}{l}\text { Mov } \\
\text { "/min }\end{array}$ & $\begin{array}{c}\text { Exp } \\
{[\mathrm{s}]}\end{array}$ & Fltr & $N_{1}$ & $\mathrm{~N}_{2}$ \\
\hline \multirow[t]{4}{*}{$2006 \mathrm{XY}$} & $2007-01-12$ & $22: 01-22: 31$ & 1.0376 & 0.0733 & 47.3 & 153.5 & -32.2 & 20.2 & 0.8 & 60 & $\bar{V}$ & 27 & 27 \\
\hline & 2007-01-16 & $21: 47-22: 27$ & 1.0433 & 0.0844 & 43.4 & 153.7 & -30.8 & 20.4 & 0.8 & 30 & V & 54 & 0 \\
\hline & 2007-01-17 & $21: 48-22: 24$ & 1.0463 & 0.0872 & 42.5 & 153.6 & -30.5 & 20.4 & 0.8 & 30 & V & 49 & 48 \\
\hline & 2007-01-19 & $21: 23-22: 18$ & 1.0526 & 0.0928 & 40.6 & 153.5 & -29.9 & 20.5 & 0.8 & 30 & V & 74 & 59 \\
\hline 2007 YH14 & 2007-01-28 & $25: 52-26: 51$ & 1.0822 & 0.1172 & 32.1 & 155.6 & -24.6 & 20.1 & 3.3 & 30 & V & 78 & 35 \\
\hline 2007 DD & 2007-02-21 & $22: 17-23: 03$ & 1.0069 & 0.0183 & 12.4 & 165.0 & -3.6 & 17.8 & 8.5 & 10 & V & 111 & 94 \\
\hline \multirow[t]{5}{*}{2007 DX } & 2007-03-18 & $20: 08-21: 12$ & 1.2074 & 0.2154 & 9.3 & 183.2 & -10.0 & 20.5 & 1.7 & 60 & $V$ & 50 & 48 \\
\hline & & 24:32-24:50 & 1.2075 & 0.2155 & 9.3 & 183.3 & -10.1 & 20.5 & 1.7 & 60 & $V$ & 15 & 0 \\
\hline & 2007-03-19 & 20:29-21:08 & 1.2080 & 0.2159 & 9.4 & 183.8 & -10.3 & 20.5 & 1.7 & 60 & $V$ & 32 & 29 \\
\hline & & $24: 29-25: 29$ & 1.2081 & 0.2160 & 9.5 & 183.9 & -10.5 & 20.5 & 1.7 & 60 & V & 49 & 48 \\
\hline & 2007-03-20 & $20: 33-21: 03$ & 1.2087 & 0.2164 & 9.6 & 184.3 & -10.8 & 20.5 & 1.6 & 30 & $C$ & 41 & 21 \\
\hline \multirow[t]{2}{*}{2007 HD84 } & 2007-05-11 & $23: 36-24: 32$ & 1.1372 & 0.1301 & 11.8 & 217.7 & 1.2 & 20.1 & 3.6 & 30 & V & 74 & 72 \\
\hline & 2007-05-12 & $23: 52-24: 36$ & 1.1378 & 0.1304 & 11.4 & 219.0 & 0.5 & 20.1 & 2.5 & 30 & V & 57 & 34 \\
\hline $2007 \mathrm{KE}$ & 2007-05-23 & $21: 26-23: 42$ & 1.1382 & 0.1379 & 22.9 & 241.1 & 25.9 & 19.6 & 5.3 & 30 & V & 159 & 88 \\
\hline 2007 KE4 & 2007-05-27 & 20:19-21:10 & 1.0303 & 0.0181 & 19.6 & 262.2 & -12.0 & 17.5 & 44.1 & 2 & $C$ & 119 & 97 \\
\hline 2007 LT & 2007-06-15 & 27:00-27:57 & 1.0842 & 0.0845 & 34.6 & 300.6 & 6.1 & 18.7 & 2.5 & 15 & V & 114 & 58 \\
\hline 2007 LW19 & 2007-06-18 & 20:08-21:13 & 1.0418 & 0.0447 & 53.8 & 213.5 & -18.3 & 18.8 & 7.6 & 10 & $V$ & 109 & 75 \\
\hline 2007 RS146 & 2007-09-19 & $25: 49-26: 27$ & 1.1157 & 0.1178 & 18.1 & 5.7 & -18.2 & 19.9 & 2.3 & 15 & V & 57 & 57 \\
\hline 2007 TU18 & 2007-10-12 & 22:33-23:42 & 1.0465 & 0.0487 & 4.4 & 14.7 & -0.9 & 18.8 & 10.7 & 10 & V & 124 & 112 \\
\hline \multirow[t]{2}{*}{2007 VV83 } & 2007-11-16 & 23:16-23:56 & 1.0237 & 0.0474 & 41.7 & 19.9 & -28.6 & 19.8 & 8.6 & 10 & V & 89 & 87 \\
\hline & 2007-11-17 & $23: 25-23: 57$ & 1.0224 & 0.0489 & 45.3 & 18.0 & -31.5 & 20.0 & 8.0 & 5 & V & 92 & 83 \\
\hline 2007 XO3 & 2007-12-13 & $24: 17-25: 11$ & 1.1162 & 0.1509 & 27.1 & 81.0 & -31.1 & 20.3 & 4.3 & 20 & $V$ & 94 & 64 \\
\hline \multirow[t]{2}{*}{2008 DG4 } & 2008-02-28 & $21: 52-23: 23$ & 1.0751 & 0.0853 & 7.9 & 168.1 & -1.7 & 19.1 & 2.2 & 10 & $V$ & 171 & 130 \\
\hline & 2008-03-02 & $22: 49-23: 37$ & 1.0984 & 0.1077 & 6.3 & 169.7 & -0.3 & 19.6 & 1.3 & 15 & V & 81 & 0 \\
\hline
\end{tabular}

$r$ and $\Delta$ are the distances of the asteroid from the Sun and the Earth, respectively, $\alpha$ is the solar phase angle, while $\lambda$ and $\beta$ are the geocentric, ecliptic (J2000) longitude and latitude.

then we risk being in error only when the asteroid spin axis is not far from the $P A B$, which is a relatively rare situation in the case of high amplitude objects.

During the analysis of data we encountered an ambiguity when folding two individual lightcurves, obtained at different moments in time. This could happen, for example, in the case of a short-period asteroid observed during both the east and the west track or in the case of the object, for which data were obtained on two consecutive nights. To describe this problem let us assume $N$ is a number of asteroid rotations between two corresponding features on both lightcurves, separated by the time interval $\Delta t . P$ is the period obtained as a weighted mean from individual solutions, derived for each of the two lightcurves that should be folded, and $\Delta P$ is the maximum period uncertainty (which can, for example, be equal to $3 \sigma$ ). Obviously, $N=\Delta t P^{-1}$ and, from the first derivative with respect to $P,|\Delta N| \approx \Delta t P^{-2} \Delta P$. Assuming the analyzed lightcurves have maximum signal in the second harmonic (which is most typical), and we try to match the corresponding maxima (or minima) of brightness, we should be able to do this if $\Delta N<0.5$, which in turn translates to $\Delta t<0.5 P^{2} \Delta P^{-1}$. Sometimes the asymmetries between the lightcurve extrema are masked by the noise and/or a changing geometry of observation, in which case the time interval $\Delta t$ is two times shorter:

$\Delta t<0.25 P^{2} \Delta P^{-1}$

The lightcurve maximum amplitude is often used to estimate the asteroid elongation. First, using the amplitude-phase relation (APR, Zappala et al. 1990) it is reduced to zero solar phase angle and then, assuming the asteroid is a triaxial ellipsoid of semiaxes $a>b>c$, rotating about $c$, and observed at the aspect angle of $90^{\circ}$, the $a / b$ ratio can easily be computed under the assumption of the geometric scattering. There are two caveats to such an approach: first the $m$ parameter in the APR depends on an unknown obliquity angle $\gamma$ (Gutiérrez et al. 2006) and second, the lightcurve amplitude at zero phase angle depends on the unknown aspect angle $\theta$. Both $\gamma$ and $\theta$ together with the solar phase angle $\alpha$ define the geometry of viewing/illumination, which is unknown as long as the asteroid spin axis remains unknown. We can derive, however, the minimum value for the $a / b$ ratio assuming $\theta=90^{\circ}$ and $\gamma=0^{\circ}$. The first condition is trivial and the latter, according to Gutiérrez et al. (2006), results in the maximum value of $m=0.03 \mathrm{mag} /{ }^{\circ}$. As a result we obtain the following relation for $a / b$ :

$\frac{a}{b} \geq 10^{0.4 A(\alpha) /(1+0.03 \alpha)}$

where $A(\alpha)$ is a peak-to-peak amplitude observed at a phase angle $\alpha$.

\section{Lightcurves of 14 asteroids}

In this section we will present lightcurves of the observed fastrotating asteroids, deriving their periods and elongations. We will spend more time on the first object, 2007 DX, to show the method of analysis and provide a more condensed description for the rest of the asteroids .

The aspect data and the observing log are provided in Table 1. For each night a date is given in the order of year-monthday, followed by the UTC observing time (to avoid ambiguity times after midnight are given as numbers greater than 24). In the next columns $r$ and $\Delta$ are the distances of the asteroid from the Sun and the Earth, respectively, $\alpha$ is the solar phase angle, while $\lambda$ and $\beta$ are the geocentric, ecliptic (J2000) longitude and latitude - the last five values are given for the middle of the observing interval. In the next column an average brightness $V$ of the asteroid, as predicted by the Horizons ephemeris, is given. Starting from the tenth column, the table gives the asteroid movement on 
the sky (Mov), the exposure time (Exp), and the filter used (Fltr; $V$ is a standard Kron-Cousins filter, while C stands for "clear" and denotes unfiltered exposures). In the last two columns the total number of CCD frames $N_{1}$ obtained for a given asteroid is shown, while $N_{2}$ denotes the number of frames used in the analysis.

\section{1. $2007 D X$}

The Amor asteroid 2007 DX was discovered on 17 Feb. 2007 by the Spacewatch sky survey. We observed it with SALT on three nights: 18, 19 and 20 Mar. 2007. On the first two nights both the east and west tracks were obtained, while on the third one the asteroid was observed only during the east track. The weather was photometric, but on the last night the IQ became bad in the middle of the track and thus we could not use the frames taken during its second part. Other details of the observations are given in Table 1.

Due to the relatively slow motion of 2007 DX on the sky the same comparison stars could be used for all measurements during a single track. For photometry we used the aperture adjusted to the IQ - its diameter varied from 6" to $10^{\prime \prime}$ - and measured both the asteroid and three to five comparison stars, depending on the night. As expected, the stray light and the lack of flat-field correction resulted in the distortion of the relative magnitudes of comparison stars in addition to the random noise. This effect never exceeded \pm 0.1 mag and was of little importance when compared to the 1 mag peak-to-peak brightness changes of the asteroid.

To derive a first approximation of the period we performed a Fourier analysis of the best quality data from the 19 Mar. west track. Results are presented in Fig. 1, which shows a plot of the $\chi^{2}$ (per degree of freedom) versus rotation frequency, obtained with the 4 th order Fourier series. We used the frequency instead of the period because the former better shows possible alias structures. There are four minima visible, at $f_{1}=2.35$, $f_{2}=3.13, f_{3}=4.70$, and $f_{4}=9.38 \mathrm{~h}^{-1}$, and the composite lightcurves, obtained for each of them, are presented in Fig. 2. The frequencies are related to one another: $f_{4}=4 f_{1}=3 f_{2}=2 f_{3}$ and even though the $f_{3}$ and $f_{4}$ minima are slightly lower (in the sense of $\chi^{2}$ value) than $f_{1}$ and $f_{2}$, this fact alone cannot be used to discard any of them. On the other hand we know most asteroid lightcurves are dominated by the second harmonic, caused by the elongation of the rotating body. Such lightcurves display two maxima and two minima per period and are similar to the one obtained for 2007 DX with the $f_{3}$ frequency.

While it is possible for bodies with significant elongation to display one maximum and one minimum lightcurve (a good example of this is 433 Eros), this can happen only at small aspect angles and a non-zero solar phase angle, and then the peak-topeak lightcurve amplitude is on the level of 0.1-0.2 mag only.

On the other hand, there are nearly spherical asteroids, which often display lightcurves of complicated shapes, with the signal located in the first, third and/or higher harmonics. Due to small elongations however, their lightcurve amplitudes are small and not as symmetric as the lightcurves obtained for 2006 DX with the $f_{1}$ and $f_{2}$ solutions.

From the above we see that the only physically meaningful solution for the period of $2007 \mathrm{DX}$ is connected with the $f_{3}=$ $4.70 \mathrm{~h}^{-1}$ frequency which, after the iteration over a smaller frequency interval, was found to be $4.696 \mathrm{~h}^{-1}$ or $0.2129 \pm 0.0005 \mathrm{~h}$.

During the nights from 18 to 20 March 2007 DX moved only about $1^{\circ}$ in the sky, which means it was observed at the same viewing/illumination geometry. Since all lighturves are

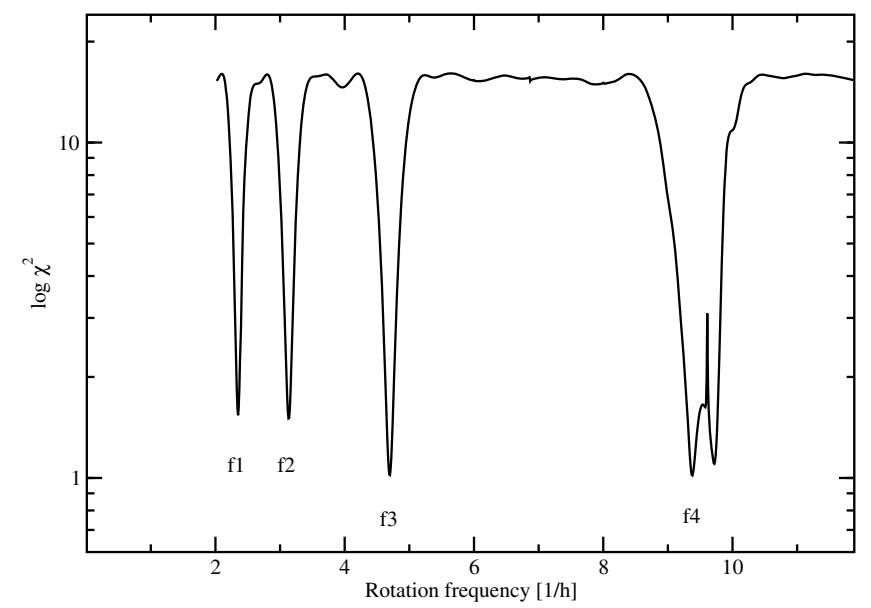

Fig. 1. $\chi^{2}$ plot for 2007 DX based on the data collected on 19 Mar. 2007 during the west track.

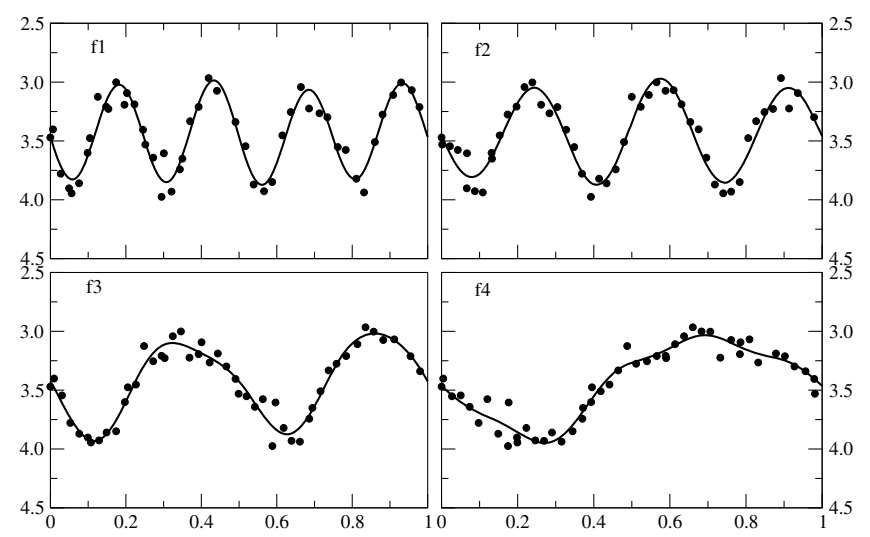

Fig. 2. Lighturves of $2007 \mathrm{DX}$ for four different rotation frequencies found in the 19 Mar. west track data.

very similar to one another we conclude 2007 DX is in the state of a relaxed rotation about the axis of maximum moment of inertia. This in turn lets us combine results from individual tracks to get a composite lightcurve and a more accurate period.

Using the obtained first approximation of the period of 2007 DX and Eq. (3), we found out that the maximum time span between two lightcurves, which could be unambiguously combined, was $\Delta t=7 \mathrm{~h}$. Fortunately, on 19 Mar. the east and west track observations were only four hours apart, so we used both data sets in a single Fourier fit, obtaining a new value for the period $P=0.21234 \pm 0.00006 \mathrm{~h}$. We proceeded this way, adding data one set at a time, until we were able to derive a unique period for all nights excluding the 18 March west track whose quality was inferior. It is $P=0.212400 \pm 0.000007 \mathrm{~h}$, and a composite lightcurve constructed with it is shown in Fig. 3 (here and in the subsequent figures, a zero phase has been corrected for light time). The lightcurve amplitude is about $1 \mathrm{mag}$, which means the minimum elongation of $2007 \mathrm{DX}$, obtained from Eq. (4), is $a / b \geq 2.0$.

\section{2. $2006 X Y$}

This asteroid is the only object in our program for which lightcurves from two different observing geometries are available. Discovered by the Catalina Sky Survey on 11 Dec. 2006 (MPEC 2006-X33), 2006 XY was first observed photometrically on 16 Dec. 2006 at Mount Lemmon Observatory. The data 


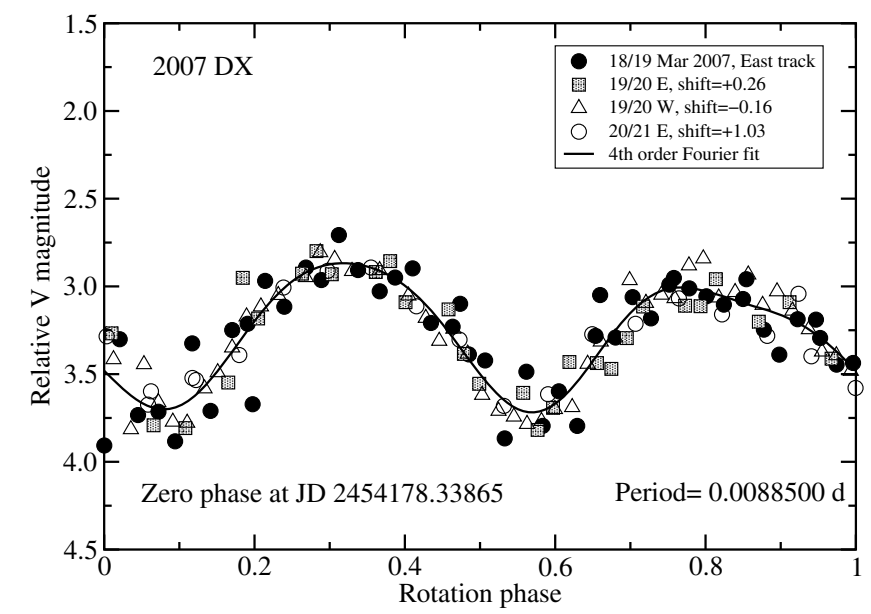

Fig. 3. A composite lightcurve of $2007 \mathrm{DX}$ obtained with a period $P=$ $0.212400 \pm 0.000007 \mathrm{~h}$.

folded with a unique period of $P=0.0830 \pm 0.0007 \mathrm{~h}$ showed an amplitude of $1.1 \mathrm{mag}$ (Hergenrother et al. 2009). We observed 2006 XY with SALT on four nights: 12, 16, 17, and 19 Jan. 2007. The images were measured with 7 " apertures, but some of them had to be discarded due to increased noise or the interference from nearby stars.

Fourier analysis of the data from single nights gave two distinct solutions for the rotation period $(0.0832 \mathrm{~h}$ and $0.1664 \mathrm{~h})$, with most of the signal in the second and the fourth harmonic, respectively. The latter gave a symmetric composite lightcurve which suggested it was an alias of the first solution. This conclusion is supported by the result of Hergenrother et al. (2009), who obtained only one solution of $=0.0830 \mathrm{~h}$.

A more accurate search for the period gave three very similar results: $0.0832 \pm 0.0002 \mathrm{~h}$ (12 Jan.), $0.0833 \pm 0.0002 \mathrm{~h}$ (17 Jan.), and $0.0831 \pm 0.0002 \mathrm{~h}$ (19 Jan.). As during that time the observing/illumination geometry did not change much (the phase angle bisector moved by only 1.5 degrees in the sky), we can conclude that the $2006 \mathrm{XY}$ is in the state of a principal axis rotation with the sidereal period of $P_{1}=0.0832 \pm 0.0001 \mathrm{~h}$. A composite lightcurve obtained with this period is presented in Fig. 4. Its amplitude of $0.8 \mathrm{mag}$ at a phase angle $\alpha=44^{\circ}$ suggests an elongation of $a / b \geq 1.4$.

Contrary to 2007 DX, a relatively long gap in time between 17 and 19 Jan. makes it impossible to unambiguously combine both nights in a simultaneous fit for the period. However, since there is still data from 12 Jan., we decided to check if it helps to limit the uncertainty in the number of asteroid revolutions. As the quality of the 12 Jan. lightcurve was lower then that from 17 and 19 Jan. we did not attempt to combine all three at once. Assuming the true period of $2006 \mathrm{XY}$ is included in the interval $P_{1}-3 \sigma, P_{1}+3 \sigma$, we first computed all possible solutions from the simultaneous 4 th order Fourier fit with the 17 and 19 Jan. data. As a result we obtained nine values of the period with the accuracy of $\sigma=0.000001 \mathrm{~h}$ each. Next we assumed that the true period is within $3 \sigma$ of each of the nine solutions, added the 12 Jan. data to the set and repeated the Fourier fit. This time we obtained only two solutions for period: $P_{2}=$ $0.0829783 \pm 0.0000003 \mathrm{~h}$ and $P_{3}=0.0831226 \pm 0.0000004 \mathrm{~h}$ (with similar $\chi^{2}$ values), which could not be refined further. As the approximate difference between synodic and sidereal periods for $2006 \mathrm{XY}$, derived with Eq. (2) is $\Delta P_{\text {syn-sid }}=0.0000002 \mathrm{~h}$,

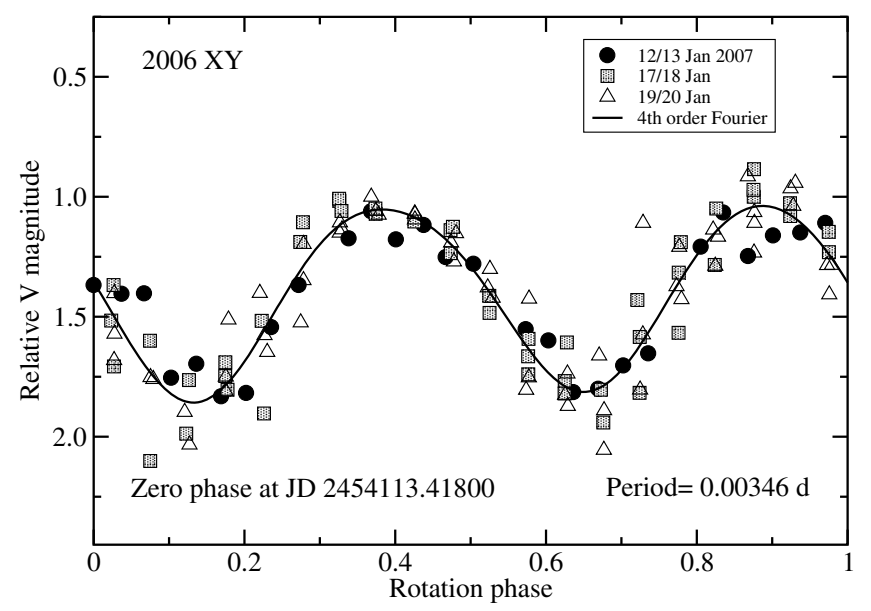

Fig. 4. Composite lightcurve of $2006 \mathrm{XY}$, obtained with a period of $0.0832 \pm 0.0001 \mathrm{~h}$.

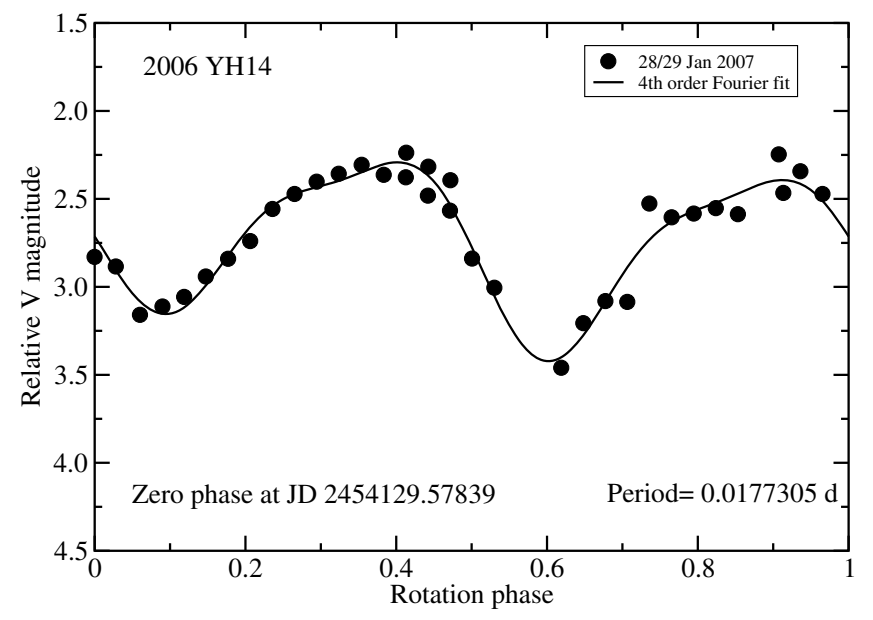

Fig. 5. Composite lightcurve of 2006 YH14 obtained with a period of $0.4251 \pm 0.0045 \mathrm{~h}$.

the obtaind results can be treated as solutions for the sidereal period of this asteroid.

\section{3. $2006 \mathrm{YH}_{14}$}

This Amor asteroid was discovered on 27 Dec. 2006 by the Catalina Sky Survey and announced in MPEC 2006-Y54. We observed it on 28 Jan. 2007, but the weather was nonphotometric, with occasionally passing clouds, therefore only half of the frames were useful. They were measured with the $7^{\prime \prime}$ diameter apertures.

A composite lightcurve obtained with a best-fit period of $P=$ $0.4251 \pm 0.0045 \mathrm{~h}$ is presented in Fig. 5. The other solutions with the maximum signal in the 3rd and 4th harmonic can be discarded based on the composite lightcurves obtained for them. A solution with half of the period given above is in principle possible but very unlikely given a large phase angle $\alpha=32^{\circ}$ during observations and a peak-to-peak amplitude of $1.1 \mathrm{mag}$. The shape elongation of this asteroid is $a / b \geq 1.7$.

\section{4. $2007 D D$}

2007 DD, a $H=25.8$ mag Aten asteroid, was discovered by the Catalina Sky Survey on 17 Feb. 2007 (MPEC 2007-D15) and observed with SALT on 21 Feb. under photometric conditions 
T. Kwiatkowski et al.: Photometric survey of NEAs with SALT. I.

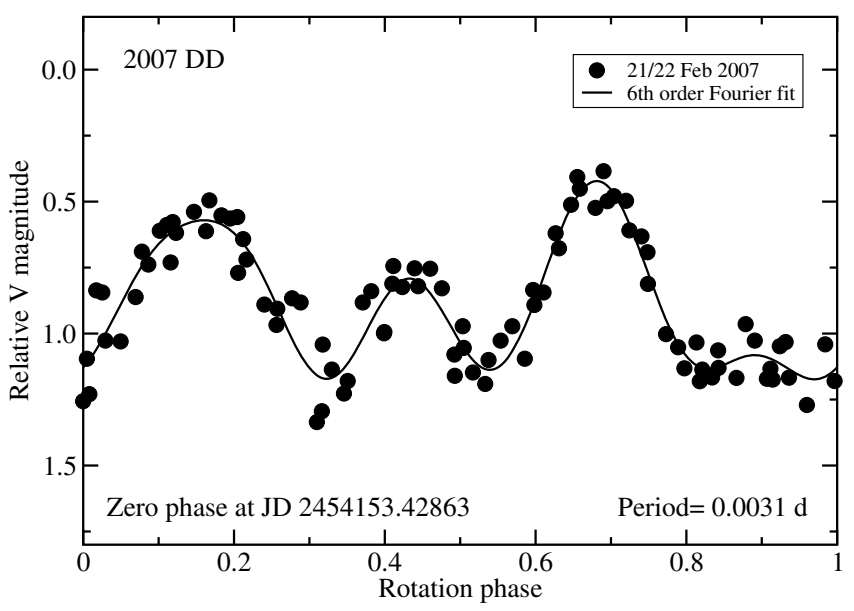

Fig. 6. Composite lightcurve of $2007 \mathrm{DD}$, obtained with a period $P=$ $0.07429 \pm 0.00007 \mathrm{~h}$.

and good seeing. Unfortunately, due to the IQ issues, a large $12^{\prime \prime}$ size aperture had to be used in the measurements. However, given the asteroid brightness of $V=17.8 \mathrm{mag}$, it did not introduce too much noise to the measurements.

A 6th order Fourier analysis of the data revealed two solutions, at $P_{1}=0.07429 \pm 0.00007 \mathrm{~h}$ and $P_{2}=0.03711 \pm 0.00006 \mathrm{~h}$ (the 4th order fit gave very similar results) with the $\chi^{2}$ value for $P_{2}$ being twice as high as for $P_{1}$. A composite lightcurve obtained for the first solution is presented in Fig. 6 and is dominated by the second harmonic with significant signal also in the 1 st and 3rd harmonics. Contrary to this, a composite lightcurve plotted with $P_{2}$ is dominated by the 1 st harmonic, with some signal also in the 2 nd harmonic. Taking all this into account we prefer the first solution, $P_{1}$.

A suspicious flat minimum between phases 0.8 and 1.0 (Fig. 6) is composed of points obtained throughout the whole observing run, so we believe it is a true feature. In fact, a similar minimum was observed in the lightcurve of another small, fast rotation asteroid, $1999 \mathrm{SF}_{10}$ (Pravec et al. 2000). A large lightcurve amplitude of $0.8 \mathrm{mag}$ at a relatively small phase angle suggests a significant asteroid elongation of $a / b \geq 1.7$.

\section{5. $2007 H D_{84}$}

This asteroid was discovered by the Mt. Lemmon Survey (MPEC 2007-H76) on 26 Apr. 2007. We observed it on two nights: 11 and 12 May 2007 under photometric conditions. The images were measured with a $4 . .5$ aperture, but due to the IQ issues not all points could be used.

A 4th order Fourier series fit to the 11 May data revealed a period of $P_{1}=0.2754 \pm 0.0007 \mathrm{~h}$ with most of the signal in the second and the fourth harmonic (Fig. 7). There is another possible solution at $P_{2}=0.1381 \pm 0.0004$ with similar $\chi^{2}$ value which cannot be rejected.

As the data from 12 May were of lower quality, we used only the middle part of the original lightcurve and, since there was a clear linear trend in it, we corrected it assuming the lower minima should be on the same level. A 4th order Fourier fit gave us two solutions for the period, similar to those from 11 May.

Finally we tried to perform a simultaneous fit to the data from both nights even though the condition set in Eq. (3) was not fulfilled. In the process the lightcurve from 12 May was being adjusted in magnitude. To help resolve the ambiguity in the number of cycles between both lightcurves we assumed the true

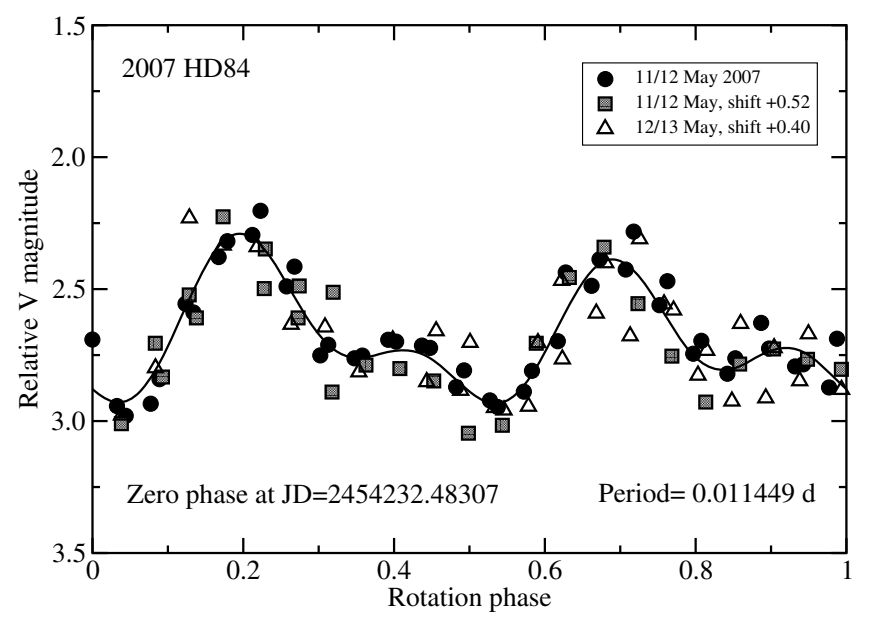

Fig. 7. Composite lightcurve of 2007 HD84, obtained with a period $P=$ $0.2754 \pm 0.0007 \mathrm{~h}$.

period should be bracketed in the interval $P_{1}-3 \sigma, P_{1}+3 \sigma$. This restriction limited the number of solutions to just three: $0.27323,0.27478,0.27323 \mathrm{~h}$ (all with $\sigma=0.00002 \mathrm{~h}$ ), with the middle one being the most probable, as it is only $1 \sigma$ from $P_{1}$. In the case of $P_{2}$, we obtain: $0.137389,0.138167,0.138958 \mathrm{~h}$ $(\sigma=0.000008 \mathrm{~h})$ and again, the middle value is the most probable.

The composite lightcurve, obtained with $P_{1}$ is presented in Fig. 7. Its maximum amplitude is $0.7 \mathrm{mag}$, which translates to an elongation of $a / b \geq 1.6$.

\section{6. $2007 \mathrm{KE}$}

$2007 \mathrm{KE}$, a $H=22.5 \mathrm{mag}$ asteroid, was discovered on 16 May 2007 by the Catalina Sky Survey (MPEC 2007-K06). We observed it on 23 May - at that time its declination was about $\delta=5^{\circ}$, and because of that SALT could follow it for more then two hours. Unfortunately, the weather was not good, with thin cirrus covering the sky.

Due to the FoV shift in the middle of the track, the frames were split into two parts, both measured with the $8^{\prime \prime}$ apertures but referred to different comparison stars. The 4th order Fourier fit to the data revealed a period of $0.731 \pm 0.003 \mathrm{~h}$. A composite lightcurve of $2006 \mathrm{KE}$ (Fig. 8) presents an amplitude of 0.9 mag, which suggests the asteroid elongation of $a / b \geq 1.6$.

\section{7. $2007 K E_{4}$}

$2007 \mathrm{KE}_{4}$ was an exceptional object in our program due to its very fast movement on the sky $\left(44^{\prime \prime} / \mathrm{min}\right)$. Discovered on 24 May 2007 by the Catalina Sky Survey, this small $H=$ $25.2 \mathrm{mag}$ asteroid passed the Earth on 27.9 May at a distance of $\Delta=0.0181 \mathrm{AU}$ (MPEC 2007-K44). On that night it was $62^{\circ}$ from the Moon (the phase of which was 0.85 ), and due to the altitude limits of SALT we could observe it only in the bright time. Fortunately the asteroid brightness was $V=17.5$ mag and the night was photometric, so the target stood out well above the sky background. In order not to trail its image we used a $2 \mathrm{~s}$ exposure time and a "clear" filter, with which we still had $S / N$ of 50-100 for the asteroid. Unfiltered photometry was generally problematic due to the stray light, but in this case (mainly due to the short exposure time) the effect was smaller than that of the bright background caused by the moonlight. 


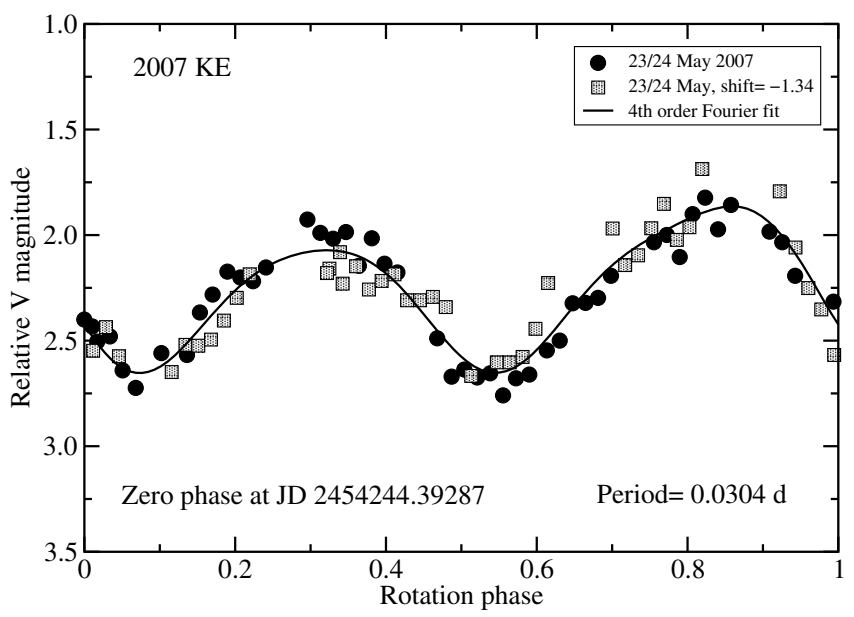

Fig. 8. Composite lightcurve of $2007 \mathrm{KE}$, obtained with a period $P=$ $0.731 \pm 0.003 \mathrm{~h}$. The second part of data marked by squares is of much lower accuracy and was used only to derive a more accurate value for the period (due to the extended time coverage). A 4th order Fourier fit, approximating the lightcurve, was obtained only from the first part of the data (circles)

The images were dominated by a radial decrease of the background level caused by the optical system. Because of that it we created night sky flat-fields by first removing stars from several dithered images and then median-combining them.

After the flat-field correction the asteroid and several comparison stars were measured with $6^{\prime \prime}-9^{\prime \prime}$ apertures. On many occasions the target was too close to a star or a bad pixel/column, so not all images were useful. Because of the lack of common comparison stars we divided the whole data set into five series, which had to be adjusted in magnitude.

An initial $\chi^{2}$ search for the period in each of the five series separately gave the same global solution of about $77 \mathrm{~s}$, with a less likely secondary solution of about $154 \mathrm{~s}$. Composite lightcurves obtained with the latter, however, gave four maxima and minima per period, and the curve was a symmetric multiplication of the $77 \mathrm{~s}$ solution. Because of that we prefer the shorter period, even though the longer one cannot be rejected.

A search for a period with all five series of data, with simultaneous adjustment in magnitude, gave a solution of $P=$ $0.021408 \pm 0.000007 \mathrm{~h}(77.1 \mathrm{~s})$ and a composite lightcurve shown in Fig. 9. The lightcurve amplitude of 0.4 mag translates to the elongation of $2007 \mathrm{KE}_{4}$ of $a / b \geq 1.3$.

In June 2007 there was only one asteroid known with a similarly short period $-2000 \mathrm{DO}_{8}-$ which rotates once every $78.2 \mathrm{~s}$ (Whiteley et al. 2002b). Currently an even faster spinning object is known, $2008 \mathrm{HJ}$, whose period is only $42 \mathrm{~s}$ (Miles 2008).

\section{8. $2007 L T$}

Discovered by the Catalina Sky Survey on 9 Jun. 2007 (MPEC 2007-L39) this Apollo asteroid was observed with SALT on 15 Jun. The images were measured with a $7^{\prime \prime}$ aperture. In the middle of the one hour track there was passing cirrus which increased the noise in the data, and so only the first and the last part of the lightcurve were used in the Fourier analysis.

The search for a period with the 6th order Fourier series, with the second part of lightcurve being shifted in magnitude, revealed a global solution at $P=0.044583 \pm 0.000022 \mathrm{~h}$ with a second possibility at $2 P$. The latter, however, is unrealistic as it

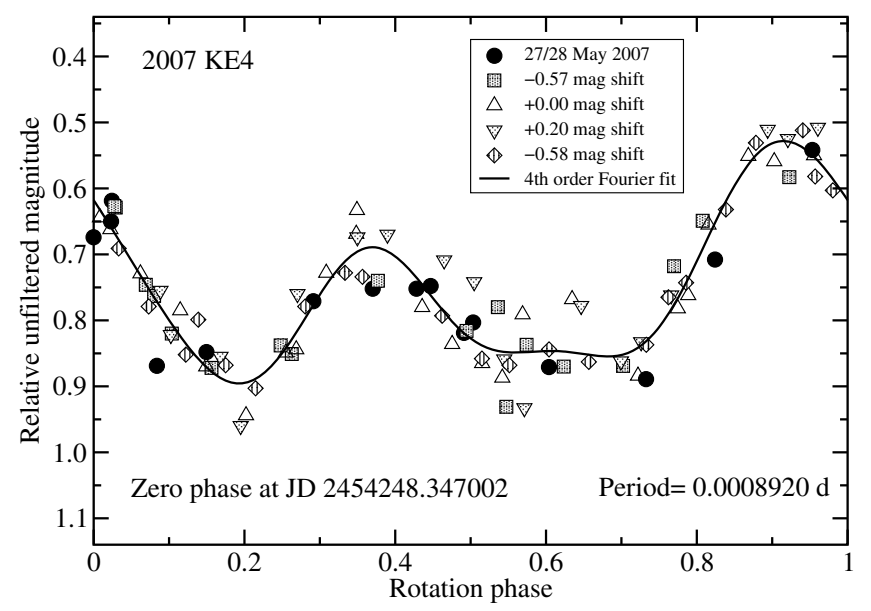

Fig. 9. Composite lightcurve of $2007 \mathrm{KE} 4$ obtained with a period of $P=0.021408 \pm 0.000007 \mathrm{~h}$.

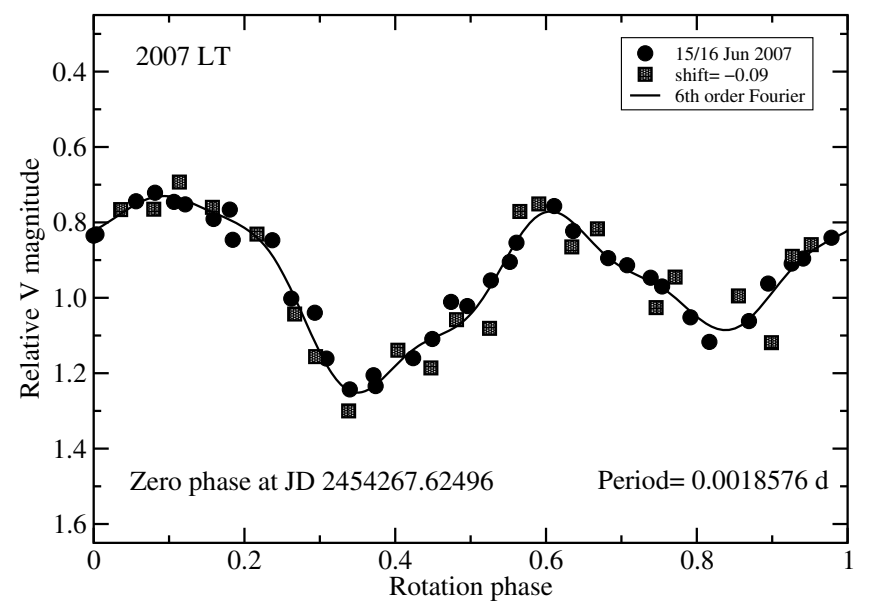

Fig. 10. Composite lightcurve of 2007 LT obtained with a period of $P=0.044583 \pm 0.000022 \mathrm{~h}$.

contains most of the signal in the 2 nd and 4th harmonic, and the composite lightcurve obtained with it is a symmetric multiplication of the lightcurve of the shorter period solution, presented in Fig. 10. The elongation of this asteroid, based on its lightcurve amplitude of $0.5 \mathrm{mag}$, is $a / b \geq 1.3$.

\section{9. $2007 L W_{19}$}

2007 LW $_{19}$ was discovered by the Siding Spring Survey on 15 Jun. 2007 (MPEC 2007-M06) and observed with SALT on 18 Jun.

Despite the photometric weather, instrumental problems interfered during observations, and some images had to be discarded. The rest were measured with an aperture of $8^{\prime \prime}$ in diameter. A search for the period showed a global minimum at $P=0.10169 \pm 0.00014 \mathrm{~h}$ with a clear alias at $2 P$.

A composite lightcurve (Fig. 11) has a peak-to-peak amplitude of 0.6 mag which, after the correction to a zero solar phase angle, suggests the asteroid axis ratio $a / b \geq 1.2$.

\subsection{0. $2007 R S_{146}$}

$2007 \mathrm{RS}_{146}$ is the second-fastest rotating asteroid reported in this paper. It was a Catalina Sky Survey discovery from 15 Sep. 2007 (MPEC 2007-S13), observed by us under clear skies on 19 Sep. 


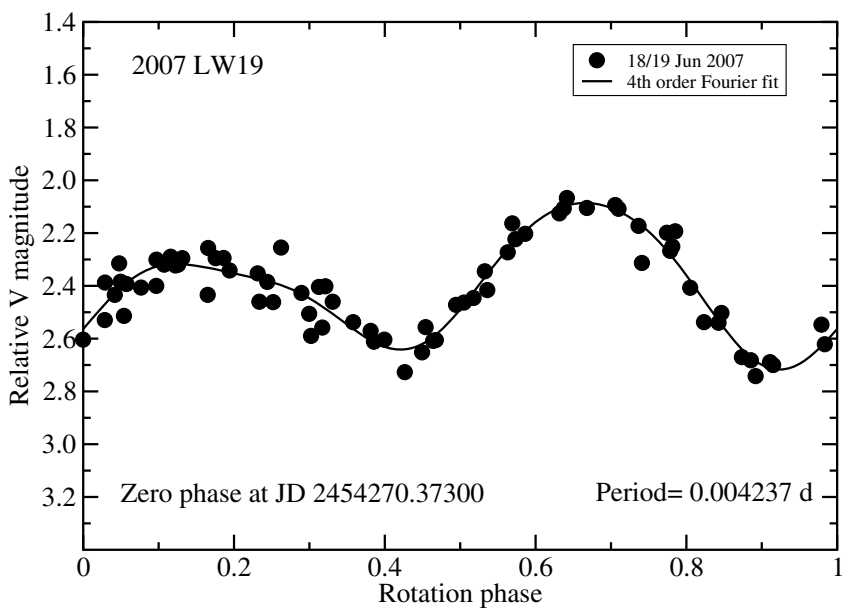

Fig. 11. Composite lightcurve of 2007 LW19 obtained with a period of $P=0.10169 \pm 0.00014$ h.

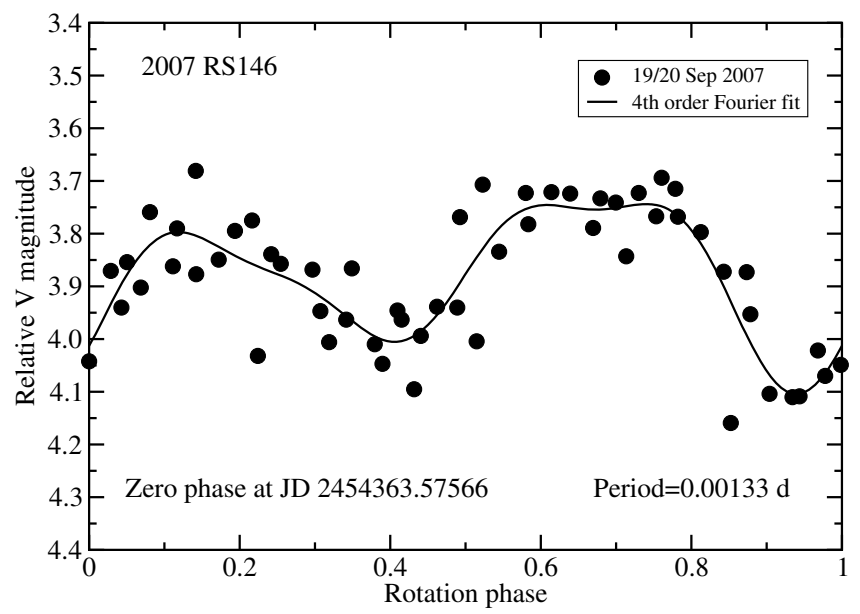

Fig. 12. Composite lightcurve of $2007 \mathrm{RS}_{146}$ obtained with a period of $P_{1}=0.03209 \pm 0.00004 \mathrm{~h}$.

The images were obtained with a smaller pixel size of $0.23(2 \times 2$ binning), which increased the read-out time.

The asteroid and several stars were measured with a 5.'5 aperture. A 4th order Fourier fit gave two solutions for the rotation period: $P_{1}=0.03209 \pm 0.00004 \mathrm{~h}$ and $P_{2}=0.0642 \pm$ $0.0001 \mathrm{~h}$ with the maximal signal in the second and fourth harmonic, respectively. The composite lightcurve obtained for $P_{2}$ is not a symmetric multiplication of the first solution lightcurve (as it is the case among other asteroids in this paper) and is equally likely.

The composite lightcurve obtained with $P_{1}$ is presented in Fig. 12. Its amplitude of 0.4 mag suggests a shape elongation of $a / b \geq 1.3$.

\subsection{1. $2007 T U_{18}$}

Discovered on 9 Oct. 2007 by LINEAR (MPEC 2007-T61), $2007 \mathrm{TU}_{18}$ was observed by SALT on 12 Oct. under photometric conditions. The CCD frames were measured with the $6^{\prime \prime}$ apertures, but some of them were unusable either due to the asteroid passing a bright star or the IQ issues. As the FoV was shifted once during the track, part of the lightcurve had to be adjusted in magnitude during the Fourier analysis of the data. There were four solutions found for the period, with the maximum signal in the first, second, third and the fourth harmonic,

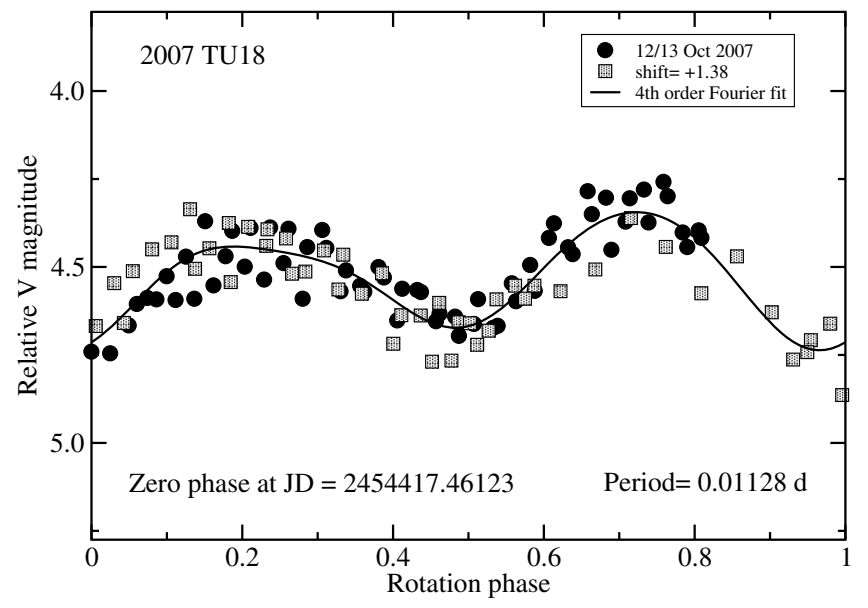

Fig. 13. Composite lightcurve of $2007 \mathrm{TU}_{18}$ obtained with a period of $P=0.271 \pm 0.001 \mathrm{~h}$.

and due to the lightcurve noise all of them are generally possible. However, the small solar phase angle of $4^{\circ}$ during observations suggests the two maxima, two minima lightcurve obtained with $P=0.271 \pm 0.001 \mathrm{~h}$ (see Fig. 13) is the most probable, with the period twice as long also possible. The lightcurve peak-to-peak amplitude of 0.5 mag suggests 2007 TU18 has an elongation of $a / b \geq 1.5$.

\subsection{2. $2007 V V_{83}$}

This asteroid was discovered on 7 Nov. 2007 by LINEAR (MPEC 2007-V64) and observed with SALT on 16 Nov. during photometric weather. The images were reduced with a 5" diameter aperture. The last 18 points on the lightcurve were measured with a different comparison star and were adjusted in magnitude during a 4 th order Fourier fit. The rotation period found is $P_{1}=0.15370 \pm 0.00053 \mathrm{~h}$.

The observations from 17 Nov. are much noiser because a shorter exposure time was mistenkenly used. The rotation period found in the data is $P_{2}=0.15446 \pm 0.00082 \mathrm{~h}$, in good agreement with $P_{1}$. The weighted average from the two nights is $P=0.15392 \pm 0.00045 \mathrm{~h}$, and this accuracy is not sufficient to combine them in a simultaneous fit (to be able to do that, both lightcurves would have to be obtained within a time span of $\Delta t \leq 4 \mathrm{~h}$ ).

A composite lightcurve of $2007 \mathrm{VV}_{83}$ obtained with the average period is shown in Fig. 14. The lightcurve peak-to-peak amplitude is $0.9 \mathrm{mag}$, and the asteroid shape has elongation of $a / b \geq 1.4$.

\subsection{3. $2007 \mathrm{XO}_{3}$}

On 4 Dec. 2007, the Catalina Sky Survey discovered a $H=$ 22.9 mag asteroid, which displayed a brightness change of $1.5 \mathrm{mag}$ within $10 \mathrm{~min}$, suggesting it might be a fast rotator. In MPEC 2007-X21 it received a designation $2007 \mathrm{XO}_{3}$.

We observed this asteroid on 13 Dec. 2007 under photometric conditions. The last part of the 55 min track, however, had to be discarded due to the inferior IQ. The remaining frames were measured with a $5^{\prime \prime}$ diameter aperture. A 4th order Fourier fit to the data gave one solution at $P=0.2530 \pm 0.0015 \mathrm{~h}$. There were also three other local minima on the $\chi^{2}$ plot with a maximum signal in the 1 st, 3 rd and 4th harmonic, but when used to 


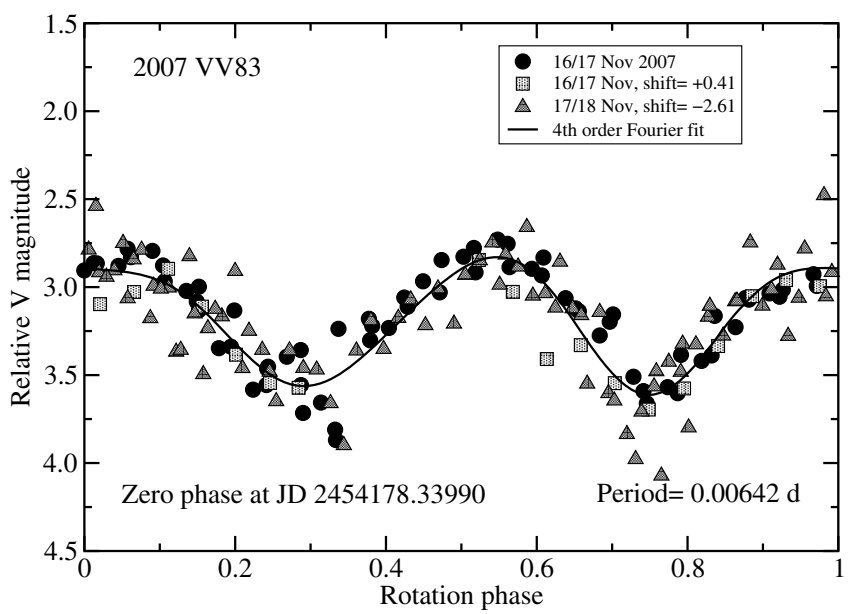

Fig. 14. Composite lightcurve of 2007 VV83 obtained with a period of $P=0.15392 \pm 0.00045 \mathrm{~h}$.

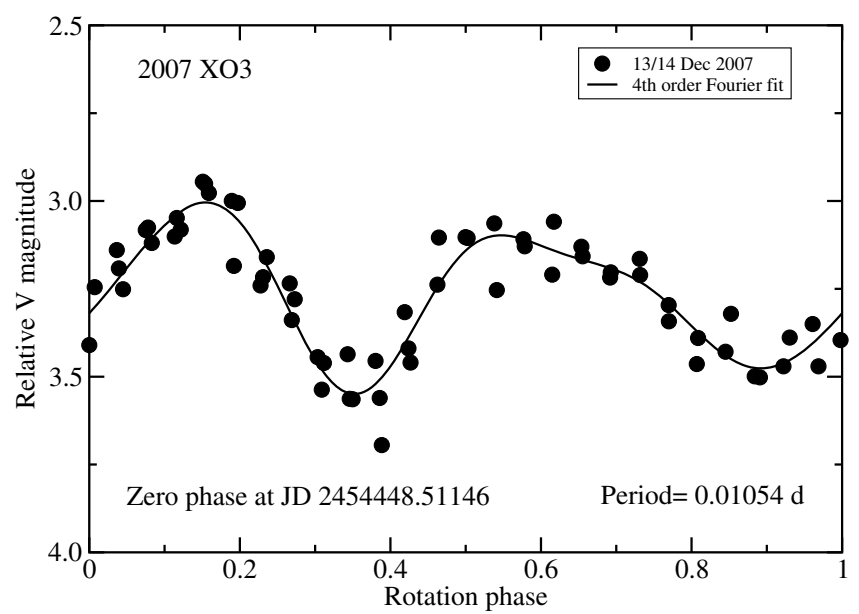

Fig. 15. Composite lightcurve of $2007 \mathrm{XO} 3$ obtained with a period of $P=0.2530 \pm 0.0015 \mathrm{~h}$.

fold the data they gave clearly inferior results, so we can safely accept the global minimum as the true one.

A composite lightcurve of $2007 \mathrm{XO}_{3}$ is shown in Fig. 15. The amplitude is about $0.6 \mathrm{mag}$, and the corresponding shape elongation of this asteroid is $a / b \geq 1.4$.

\subsection{4. $2008 D G_{4}$}

This Apollo asteroid was discovered on 27 Feb. 2007 by the LONEOS survey (MPEC 2008-D49) and observed by us on 28 Feb. and 2 Mar., during photometric conditions.

The images obtained on 28 Feb. were measured with a $5^{\prime \prime}$ diameter aperture and revealed easily visible quasi-sinusoidal brightness changes with a linear trend of $2.95 \mathrm{mag} \cdot \mathrm{d}^{-1}$ superimposed. The comparison stars showed similar effects, but with different slopes. After removing the trend, a solution for a period was found at $P_{1}=0.2179 \pm 0.0005 \mathrm{~h}$. A composite lightcurve obtained for it is presented in Fig. 16.

The data collected on 2 Mar. were measured with a 4 " aperture, but the lightcurve was more noisy than on 28 Feb., which resulted in a lower accuracy of the derived period. The obtained value $P_{2}=0.2194 \pm 0.0017 \mathrm{~h}$ is consistent with $P_{1}$ derived from the 28 Feb. data within given uncertainties.

Unfortunately, a long gap of $72 \mathrm{~h}$ between both lightcurves makes it impossible to combine them in a single fit. Using

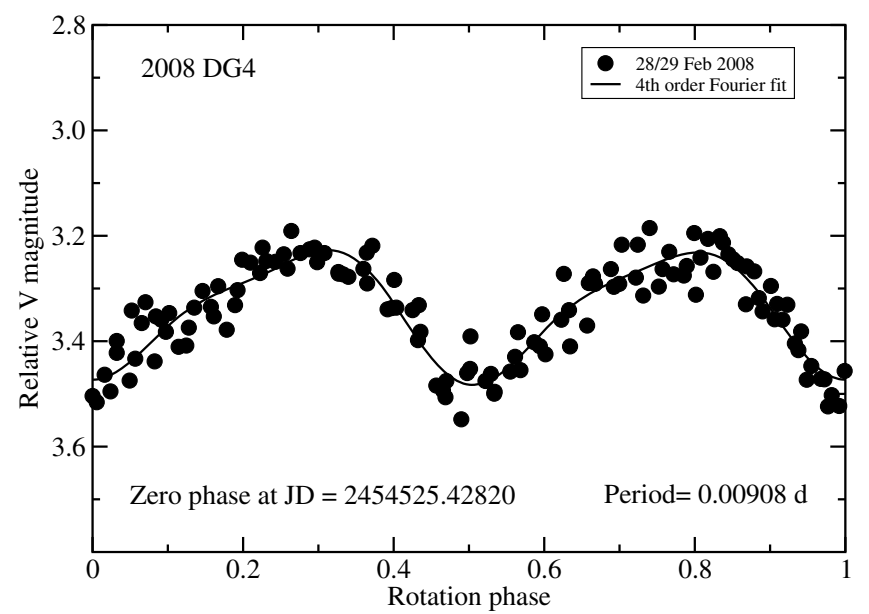

Fig. 16. Composite lightcurve of 2008 DG4 obtained with a period of $P=0.2179 \pm 0.0005 \mathrm{~h}$.

Eq. (3), assuming $P=0.2179 \mathrm{~h}$ and $\Delta P=3 \sigma_{P}=0.0015 \mathrm{~h}$, we find that to fold both lightcurves without ambiguity they should be acquired no longer than about eight hours apart.

A composite lightcurve of $2008 \mathrm{DG}_{4}$ from 28 Feb. 2007 is shown in Fig. 16. The amplitude is 0.3 mag which allows us to estimate its elongation as $a / b \geq 1.2$. The lightcurve from 2 Mar. is more noisy, but it has a similar shape and amplitude as the one in Fig. 16. Because of its lower quality we used it only as a reference and adopt $P=0.2179 \pm 0.0005 \mathrm{~h}$ as a synodic period of $2008 \mathrm{DG}_{4}$.

\section{Summary}

A summary of the results is presented in Table 2. Starting from the second column, it contains the derived synodic period of the rotation $P$, its uncertainty $\sigma_{P}$, the peak-to-peak lightcurve amplitude $A$, the estimated minimum elongation $a / b$ of the asteroid and the effective diameter $D$.

As all rotation periods reported here are very short even though during the observations the asteroid moved fast on the sky, the position of the phase angle bisector did not change much. Because of that the difference between the synodic and sidereal periods is insignificant in most cases. To check this we computed for each object in Table 2 the $\tau=\sigma_{P} / \Delta P_{\text {syn-sid }}$ ratio, where the approximate value for $\Delta P_{\text {syn-sid }}$ was obtained from Eq. (2). Only in two cases $\tau$ was smaller than 10: for $2006 \mathrm{XY}$ we obtained $\tau=1.6$, and for $2007 \mathrm{DX} \tau=1.7$. As the lightcurve amplitudes of both objects are large, it is very unlikely that their spin axes are close to the PAB, and so Eq. (2) should be a good approximation of $\Delta P_{\text {syn-sid }}$. This in turn means that even in the case of 2006 XY and 2007 DX, their periods can be regarded as close to sidereal.

Almost all our periods in Table 2 have $U=2$ which, following the criteria of Warner et al. (2009) indicates a result which may be wrong by an integer ratio. Despite this, periods with $U \geq 2$ are usually used for statistical studies of rotation rates. For $2006 \mathrm{XY}$ we have $U=3$ as its period is known without ambiguity.

The effective diameters $D$ have been derived from the absolute magnitudes $H$ available in the Horizons database. To be able to compare $D$ to the diameters provided in the LCDB we used Eq. (1) from Warner et al. (2009) in the calculations with the geometric albedo $p_{\mathrm{V}}=0.20$. 
Table 2. Summary of the results.

\begin{tabular}{llllll}
\hline \hline Asteroid & $\begin{array}{c}P \\
{[\mathrm{~h}]}\end{array}$ & $\begin{array}{c}\sigma_{P} \\
{[\mathrm{~h}]}\end{array}$ & $\begin{array}{l}A \\
{[\mathrm{mag}]}\end{array}$ & $a / b$ & $\begin{array}{l}D \\
{[\mathrm{~km}]}\end{array}$ \\
\hline 2006 XY & 0.0829783 & \pm 0.0000003 & 0.8 & 1.4 & 0.047 \\
(2nd solution) & 0.0831226 & \pm 0.0000004 & & & \\
2006 YH14 & 0.4251 & \pm 0.0045 & 1.1 & 1.7 & 0.068 \\
2007 DD & 0.07429 & \pm 0.00007 & 0.8 & 1.7 & 0.021 \\
2007 DX & 0.212400 & \pm 0.000007 & 1.0 & 2.0 & 0.082 \\
2007 HD84 & 0.2754 & \pm 0.0007 & 0.7 & 1.6 & 0.057 \\
(2nd solution) & 0.1381 & \pm 0.0004 & & & \\
2007 KE & 0.731 & \pm 0.003 & 0.9 & 1.6 & 0.094 \\
2007 KE4 & 0.021408 & \pm 0.000007 & 0.4 & 1.3 & 0.027 \\
2007 LT & 0.044583 & \pm 0.000022 & 0.5 & 1.3 & 0.094 \\
2007 LW19 & 0.10169 & \pm 0.00014 & 0.6 & 1.2 & 0.059 \\
2007 RS146 & 0.03209 & \pm 0.00004 & 0.4 & 1.3 & 0.065 \\
(2nd solution) & 0.0642 & \pm 0.0001 & & & \\
2007 TU18 & 0.271 & \pm 0.001 & 0.5 & 1.5 & 0.033 \\
2007 VV83 & 0.15392 & \pm 0.00045 & 0.9 & 1.4 & 0.033 \\
2007 XO3 & 0.2530 & \pm 0.0015 & 0.6 & 1.4 & 0.082 \\
2008 DG4 & 0.2179 & \pm 0.0005 & 0.3 & 1.2 & 0.052 \\
\hline
\end{tabular}

$P$ is the synodic period, $A$ is the lightcurve amplitude, $a / b$ is the minimum elongation and $D$ is the effective diameter.

\section{Conclusions}

This is the first paper in the series presenting results of the photometric survey of the very small asteroids, in which we focused on the fast-rotating objects. We presented lightcurves of 14 asteroids, deriving their rotation periods and shape elongations. The shortest period object in our sample is $2007 \mathrm{KE}_{4}$, which rotates every $77 \mathrm{~s}$, and the longest period of $44 \mathrm{~min}$ was found for $2007 \mathrm{KE}$. Due to the observing limitations (we were unable to detect lightcurves of amplitudes smaller than about $0.2 \mathrm{mag}$ ), most presented asteroids have large amplitudes and have significant elongations. Their fast spins are most probably caused by the YORP effect, which we will discuss in more detail in the next paper in the series. There we will also show which of the presented asteroids will be accessible for further studies in the near future.
Acknowledgements. T.K. is grateful to A. Kryszczynska, M. Polinska and E. Bruss-Kwiatkowska for their help in reduction of some of data. All of the observations reported in this paper were obtained with the Southern African Large Telescope (SALT), a consortium consisting of the National Research Foundation of South Africa, Nicholas Copernicus Astronomical Center of the Polish Academy of Sciences, Hobby Eberly Telescope Founding Institutions, Rutgers University, Georg-August-Universitat Gottingen, University of Wisconsin-Madison, Carnegie Mellon University, University of Canterbury, United Kingdom SALT Consortium, University of North CarolinaChapel Hill, Dartmouth College, American Museum of Natural History and the Inter-University Centre for Astronomy and Astrophysics, India.

\section{References}

de Luise, F., Perna, D., Dotto, E., et al. 2007, Icarus, 191, 628

Dermawan, B. 2004, Ph.D. Thesis, School of Science, Univ. of Tokyo

Gutiérrez, P. J., Davidsson, B. J. R., Ortiz, J. L., Rodrigo, R., \& Vidal-Nuñez, M. J. 2006, A\&A, 454, 367

Hergenrother, C., \& Whiteley, R. J. 2005, BAAS, 37, 636

Hergenrother, C. W., Whiteley, R. J., \& Christensen, E. J. 2009, Minor Planet Bulletin, 36, 16

Hicks, M., Lawrence, K., Rhoades, H., et al. 2009, The Astronomer's Telegram, 2116,1

Holsapple, K. A. 2007, Icarus, 187, 500

Jenniskens, P., Shaddad, M. H., Numan, D., et al. 2009, Nature, 458, 485

Kwiatkowski, T., Kryszczynska, A., Polinska, M., et al. 2009, A\&A, 495, 967

Masiero, J., Jedicke, R., Ďurech, J., Gwyn, Denneau, L., \& Larsen, J. 2009, Icarus, 204, 145

Miles, R. 2008, The Astronomer, 45, 43

Ostro, S. J., Giorgini, J. D., Benner, L. A. M., et al. 2003, Icarus, 166, 271

Ostro, S. J., Pravec, P., Benner, L. A. M., et al. 1999, Science, 285, 557

Pravec, P., Harris, A. W., \& Michalowski, T. 2002, in Asteroids III, ed. W. F. B. Jr., A. Cellino, P. Paolicchi, \& R. P. Binzel (Tucson: University of Arizona Press), 113

Pravec, P., Harris, A. W., Scheirich, P., et al. 2005, Icarus, 173, 108

Pravec, P., Hergenrother, C., Whiteley, R., et al. 2000, Icarus, 147, 477

Saito, J., Miyamoto, H., Nakamura, R., et al. 2006, Science, 312, 1341

Steel, D. I., McNaught, R. H., Garradd, G. J., Asher, D. J., \& Taylor, A. D. 1997, Planetary and Space Science, 45, 1091

Whiteley, R. J., Hergenrother, C. W., \& Tholen, D. J. 2002a, in Proceedings of Asteroids, Comets, Meteors, ed. B. Warmbein, ESA SP-500 (Noordwijk, Netherlands), 473

Whiteley, R. J., Tholen, D. J., \& Hergenrother, C. W. 2002b, Icarus, 157, 139

Zappala, V., Cellino, A., Barucci, A. M., Fulchignoni, M., \& Lupishko, D. F. 1990, A\&A, 231, 548 